\title{
Cidades Inventivas no Brasil: Hierarquia e Determinantes da lnvenção
}

\author{
Inventive Cities in Brazil: \\ Hierarchy and Determinants of Invention
}

\author{
Eduardo Gonçalves* \\ Rodrigo Rodriguez** \\ Inácio Fernandes de Araújo* ** \\ Sara Monteiro dos Santos****
}

\begin{abstract}
Resumo: O artigo analisa a relação entre a escala urbana e as invenções, medidas por patentes, em uma análise comparada de dois quadriênios (2001-2004 e 2006-2009) no Brasil. Com dados do Instituto Nacional de Propriedade Industrial (Inpi), da Relação Anual de Informações Sociais (Rais), do Instituto Brasileiro de Geografia e Estatística (IBGE) e da Coordenação de Aperfeiçoamento de Pessoal de Nível Superior (Capes), o artigo explora a distribuição hierárquica e a aglomeração espacial das invenções a partir de regras de potência (lei de Zipf), além de investigar os principais determinantes do patenteamento municipal com modelos econométricos espaciais. Os resultados apontam que a escala urbana possui efeito significativo no patenteamento, assim como capacidade de PESD universitário, escolaridade dos trabalhadores e presença de setores de média-alta tecnologia.
\end{abstract}

Palavras-chave: Inovação regional. Hierarquia urbana. Patentes.

Abstract: The paper aims to analyze the relation between urban scale and inventive activities, measured by patents per capita, in two different periods (2001-2004 and 20062009) in Brazil. Based on data from the National Industrial Property Institute (Inpi), the Annual Social Information Report (Rais), The Brazilian Institute of Geography and Statistics (IBGE) and the Coordination for the Improvement of Higher Education Personnel (Capes), this paper presents the hierarchical distribution and the spatial agglomeration of inventive activities by means of power rules (Zipf Law), in addition to investigating the main determinants of patent activities in Brazilian municipalities based on spatial econometric models. The results reveal that urban scale has a significant impact on patenting activities as well as the capacity of university REDD, schooling of the workforce and the presence of medium and high technology industries.

Keywords: Regional innovation. Urban hierarchy. Patents.

\footnotetext{
* $\quad$ Doutor em Economia pela Universidade Federal de Minas Gerais (UFMG). Professor do Programa de Pós-Graduação em Economia da Universidade Federal de Juiz de Fora (UFJF). Pesquisador do CNPq. E-mail: eduardo.goncalves@ufjf.edu.br

** Doutorando em Economia pela Universidade Federal Fluminense (UFF). E-mail: rrodriguez431@ gmail.com

*** Doutor em Economia pela Universidade Federal de Juiz de Fora (UFJF). E-mail: inaciofaj@gmail. com

*** Graduação em Ciências Econômicas pela Universidade Federal de Juiz de Fora (UFJF). E-mail: sarah_gdc@hotmail.com
} 


\section{1 lntrodução}

O papel atribuído à localidade da inovação vem adquirindo relevância nos estudos de crescimento econômico, tanto em relação a países (ROMER, 1986; LUCAS, 1988) quanto a cidades e áreas metropolitanas (GLAESER, 1999; SIMMIE, 2001). Outra constatação é a concentração espacial das atividades inventivas ou inovativas, em particular nas grandes cidades e metrópoles (FELDMAN; FLORIDA, 1994). Entre as razões para se explicar a concentração urbana das inovações, há as evidências de externalidades regionais, conhecidas como economias de localização e urbanização (MARSHALL, 1982; JACOBS, 1969).

No caso das economias de urbanização, evidências empíricas apontam para os efeitos positivos de externalidades em cidades maiores e/ou densas com diferentes atividades produtivas, como as conexões entre mercados, tecnologias, firmas e redes de comunicação entre os agentes (MALECKI, 2014). De acordo com Malecki (2014), tais escalas de urbanização propiciam benefícios como: a) aumento da diversidade e escala de mercados especializados; b) aumento do volume e qualidade de troca de informação com suporte às funções de serviço intensivo em conhecimento; c) desenvolvimento de experiência de trabalho em conhecimento chave, com contribuições para mudanças técnicas; d) aumento da flexibilidade e mobilidade da força de trabalho e oportunidades de negócios.

Diante das evidências das externalidades, os modelos de crescimento econômico regional associam o crescimento à concentração de pessoas e firmas em áreas urbanas (CARLINO, 2001). O contato face a face proporcionado pela aglomeração de pessoas em centros urbanos facilita a troca de conhecimentos não codificados, tácitos, pois o processo de invenção é um processo predominantemente coletivo que envolve pesquisadores, firmas e instituições que estabelecem redes de relações sociais e econômicas (ARTHUR, 1989).

Ó hUallacháin (1999) aponta que a vantagem de grandes áreas urbanizadas em gerar invenções emerge da intensidade industrial tecnológica e da presença de pessoal qualificado. Essas áreas urbanas são locais onde as externalidades da urbanização facilitam a invenção, indicando que a atividade inventiva ocorre com intensidade mais que proporcional à escala urbana. O resultado de que as patentes, usadas como medida de invenção, concentram-se em grandes áreas metropolitanas é encontrado pelo autor a partir da análise da hierarquia de Zipf, uma medida de magnitude e classificação de distribuições.

Uma forma de analisar as externalidades advindas dos efeitos de urbanização

é a partir do conceito de hierarquias urbanas. O conceito de hierarquias urbanas 
sugere a existência de uma relação de hierarquia identificável a partir da distribuição de variáveis-chave dos espaços urbanos, como a população, emprego, educação e atividade industrial, que permitem agrupar as unidades regionais de acordo com sua escala urbana. No caso da atividade inventiva brasileira, uma forma de testar os efeitos da presença de externalidades advindas da escala urbana dos municípios é avaliar se as invenções estão associadas, de forma mais que proporcional, a grandes centros urbanos.

O objetivo deste artigo é analisar a relação entre a escala urbana, utilizando-se a variável populacional como proxy (KRUGMAN, 1996; RUIZ, 2005), e a capacidade de invenção dos municípios do Brasil em dois períodos, 2001-2004 e 2006-2009. Para se identificar as invenções, utilizam-se dados de depósitos de patentes com informações sobre a residência de inventores nos municípios brasileiros. Busca-se identificar o posicionamento hierárquico dos principais municípios brasileiros em termos de invenções, medidas por depósitos de patentes, avaliando-se também se há alguma associação espacial entre eles. Posteriormente, busca-se identificar os principais determinantes do patenteamento de cada município, utilizando-se modelos de econometria espacial.

O presente trabalho está estruturado, além desta seção introdutória, em mais quatro seções: a segunda apresenta a revisão de literatura; a terceira aborda a metodologia implementada para o presente estudo e descreve os dados utilizados; a quarta discorre sobre a estrutura da atividade tecnológica dos municípios brasileiros e os resultados econométricos; e, por fim, a quinta traça as conclusões, limitações e direcionamentos futuros.

\section{Grandes Cidades e Atividade Tecnológica: uma Breve Revisão da Lite- ratura}

$\mathrm{O}$ argumento de que as economias urbanas potencializam o processo de geração de invenções e inovações advém de diversas formulações teóricas. Em geral, a escala urbana favorece a formação de redes (networking) entre os indivíduos (BRESCHI; LISSONI, 2009; MIGUÉLEZ; MORENO, 2013), conexões entre indivíduos e firmas, trocas de experiências a partir da mobilidade (ALMEIDA; KOGUT, 1999) e spin-offs de trabalhadores (MUENDLER; RAUCH, 2012), criação de startups (BRENNER, 2004; HALL; LERNER, 2010), empreendedorismo (AUDRETS$\mathrm{CH}$; KEILBACH, 2005) e transbordamentos de conhecimento localizados não intencionais (FELDMAN; KOGLER, 2010). Do ponto de vista da firma, facilita-se a conexão entre diferentes setores e tecnologias (SCHERER, 1982; BRESCHI; LISSONI; MALERBA, 2003; GREUNZ, 2003), compartilhamento de pesquisa e desenvolvimento (PED) e contato com as universidades (FORAY; LISSONI, 2010), seja a partir da incorporação de indivíduos qualificados, seja por parcerias em pesquisas 
ou participação em congressos e eventos. Além disso, as economias urbanas são provedoras de infraestrutura essencial para a geração de conhecimento, como universidades, institutos técnicos, livrarias, bibliotecas, sistemas de tecnologia e informação (SIMMIE, 2001; FELDMAN; FLORIDA, 1994).

Feldman e Florida (1994) enfatizam que a inovação é fruto da infraestrutura técnica do território, como a concentração geográfica de PEBD industrial, PEBD universitário, setores inter-relacionados e serviços produtivos, os quais criam sinergia e se reforçam mutuamente. A presença de PEBD universitário gera transbordamentos de conhecimento que podem atrair a PED industrial (JAFFE, 1989) e formar clusters de empregos de alta tecnologia (ACS, FITZROY; SMITH, 1999). Implícito no argumento anterior está a ideia de que as empresas tenham capacidade de absorver tais conhecimentos provenientes das universidades (COHEN; LEVINTHAL, 1989).

Os transbordamentos de conhecimento localizados se baseiam na evidência de áreas urbanas que viabilizam os contatos face a face, fundamentais para a troca de conhecimento tácito. Segundo Van Oort e Lambooy (2014), o conhecimento tácito pode ser definido como conhecimento específico, de difícil transmissão entre os agentes, sendo desenvolvido por processos de "aprender fazendo" (learning-by-doing) ou "aprender usando" (learning-by-using). Isso significa que um agente é capaz de usar determinado produto ou processo, mas não tem capacidade de codificar e transferir de forma efetiva o know-how, diferentemente do que ocorre com o conhecimento exposto em um livro, uma patente, ou uma fórmula matemática. Mecanismos sociais como encontros em feiras profissionais, conferências, seminários e atividades sociais contribuem para a troca de conhecimento de conteúdo mais tácito. Ao concentrarem e facilitarem tais mecanismos, as grandes cidades favorecem a atividade tecnológica, tendo em vista que muito desta é realizada com base em conhecimento tácito.

Além de contatos face a face, que se tornam possíveis pela proximidade, externalidades também surgem da concentração de indústrias em determinada região, pois, aglomerando-se, obtêm menores custos de transporte, maior acesso ao comércio, facilidades relacionadas à distribuição e logística, além de proporcionar ambientes mais efetivos de aprendizagem e fomento de novas tecnologias, encontrar novos mercados e observar e experimentar novas formas de organização (MARSHALL, 1982; MALECKI, 2014; DURANTON; PUGA, 2004).

Jacobs (1969) argumenta que a densidade econômica urbana contribui para a concentração da atividade tecnológica porque o meio urbano denso, ao concentrar diferentes profissionais de várias áreas de conhecimento, permite fertilização cruzada de ideias, reduz o custo médio de busca de informação relevante e aumenta as oportunidades de negócios lucrativos, produzindo uma associação positiva entre urbanização e taxa de retorno esperada da invenção. Nos grandes 
centros urbanos, que contam com uma grande quantidade de serviços produtivos e de firmas fornecedoras de insumos, há maior divisão do trabalho, o que cria um ambiente propício à invenção e favorece o surgimento de novas atividades.

Marshall (1982) enfatiza o papel da comunicação de firmas do mesmo setor de atividade econômica concentradas no espaço na geração de externalidades que conduzem ao aumento de produtividade e inovação. Contudo, embora a literatura desenvolva trabalhos mostrando oposição entre as externalidades oriundas da diversidade e da especialização (BEAUDRY; SCHIFFAUEROVA, 2009), Rosenthal e Strange (2004) argumentam que o próprio Marshall soube reconhecer o valor da diversidade urbana, em termos de complementaridade e redução de risco em face de reduções de demanda. Por outro lado, Duranton e Puga (2004) apresentam argumentos teóricos de que as externalidades de diversificação seriam mais importantes para novos setores industriais, de rápido crescimento, ao passo que, para setores maduros, maior importância teriam as externalidades de especialização, por diminuírem os custos de produção.

Afirma-se que, quanto maior o tamanho das aglomerações urbanas, maiores são as possibilidades de economias externas positivas. Elas incluem maiores oportunidades de selecionar e combinar, de forma criativa, os fatores de produção, em relação às possibilidades oferecidas pelas pequenas áreas urbanas. Além do mais, serviços de negócios intensivos em conhecimento podem trazer conhecimentos externos a usuários potenciais, uma vez que geograficamente são altamente concentrados no topo da hierarquia urbana (MALECKI, 2014), que pode ser entendida como regiões de agrupamento geográfico, onde há importantes diferenças em termos de invenção e dinâmicas de crescimento entre os clusters.

Outra vantagem de grandes áreas concentradas, como cidades metropolitanas, é que, uma vez que uma nova trajetória tecnológica é estabelecida, as firmas usualmente permanecem sobre uma mesma trajetória, causando a possibilidade de declínio econômico. As cidades metropolitanas são capazes de minimizar esse risco ao promover uma abertura de trajetórias, diminuindo as incertezas presentes seja em uma invenção, em uma instabilidade de produção e mercado, seja em uma mudança de paradigma. As metrópoles propiciam um contato mais próximo entre mercado consumidor e fornecedores, permitindo mobilidade de empregados. Da mesma forma, as cidades consideradas "metrópoles internacionais" tendem a ter mais novas trajetórias tecnológicas e mais ramificações com outras metrópoles, devido à multiplicidade de contatos internacionais (redes) e à quantidade de organizações de consultoria. As redes, que transmitem conhecimento e experiência, e a abertura cultural dessas cidades geram possibilidades de entrada em novas áreas de negócios, deixando-as menos suscetíveis aos efeitos de declínio de qualquer setor antigo e mais inclinadas a abrigar novos setores em crescimento, em relação a cidades menores e menos internacionalizadas (SIMMIE, 2001). 
Existem trabalhos empíricos que buscam mensurar os efeitos da escala urbana sobre invenções utilizando informações de patentes, como os de Ó hUallacháin (1999) e Bettencourt, Lobo e Strumsky (2007). Ambos os trabalhos se utilizam de uma lei de potência conhecida como lei de Zipf para a análise de regularidades empíricas. Ó hUallachain (1999) aponta evidências da existência de externalidades da urbanização que potencializam o processo de invenção para as áreas metropolitanas dos Estados Unidos, no período de 1990 a 1996. Bettencourt, Lobo e Strumsky (2007) investigam também, para as metrópoles norte-americanas, no período de 1980 a 2001, a relação entre patenteamento e tamanho populacional. Os autores apresentam como resultado os efeitos crescentes à escala da atividade inventiva, com relação ao seu tamanho populacional.

Em função da revisão de textos realizada nesse artigo, nota-se que, em geral, a atividade tecnológica está vinculada ao centro urbano, pelas externalidades provenientes da localização de atividades econômicas de mesmo setor ou de setores diferentes, além da concentração de pessoal qualificado e capacidade de PEBD. Tais elementos da infraestrutura tecnológica urbana são considerados na próxima seção, que apresenta a metodologia do artigo.

\section{Metodologia}

Tendo como referência o trabalho de Ó hUallacháin (1999), será utilizada uma metodologia que consiste em fazer o ordenamento das maiores cidades e das principais cidades patenteadoras e a estimação de regressões por cidades, tendo em vista uma análise de ranking. Por meio da lei de Zipf, uma lei de potência, pode-se medir a hierarquia urbana da população e da invenção brasileira, identificando-se a distribuição espacial da população e das patentes classificadas pelo seu potencial de geração de atividade tecnológica. A classificação utilizada como aproximação para o potencial de inovação é a contagem de ocorrências de patentes.

Assim, uma maneira de se medir a hierarquia distributiva é estimar a inclinação de uma distribuição do tipo rank-size (classificação-tamanho), determinando coeficientes de leis de potência que são expressas como uma reta em um gráfico log-log de inclinação um. Então, uma lei de potência pode ser definida pela relação:

$$
Y=a X^{k}
$$

em que $a$ é uma constante de proporcionalidade e o expoente k é uma constante. Reescrevendo a equação, têm-se os resultados em um gráfico log-log: 


$$
\log Y_{i}=\log a+\mathrm{k} \log X_{i}+\varepsilon_{i}
$$

no qual a equação é de uma reta com coeficiente angular $k$.

Uma vez linearizada e estimada através de uma regressão linear simples, será encontrado um coeficiente que, conforme a lei de Zipf, que determina que a frequência $y$ é inversamente proporcional à sua classificação em uma tabela de frequência, o coeficiente deve ser negativo. Coeficientes próximos de -1 indicam uma alta hierarquização, e coeficientes mais próximos de zero indicam que não há uma hierarquia urbana, logo, a distribuição é normal. Além disso, o $R^{2}$ indica o quão eficiente é o ajuste linear.

Dessa forma, será elaborada uma equação na qual será possível obter uma classificação das cidades dado seu tamanho, representada por:

$$
\log \text { População }_{\mathrm{i}}=\log a+\mathrm{k} \log \text { rank população } o_{\mathrm{i}}+\varepsilon_{\mathrm{i}}
$$

na qual $i$ varia por cidades, log população é o logaritmo natural da população de uma dada cidade em um período determinado e log rank população é o logaritmo natural da classificação (ranking) de uma dada cidade em um período determinado, sendo k o parâmetro estimado. Se $\mathrm{k}>1$, as cidades do topo da distribuição são proporcionalmente maiores que os municípios da parte inferior da distribuição. Valores de $\mathrm{k}=1$ indicam que, na média, a distribuição é hierarquizada e proporcionalmente distribuída, de forma que o primeiro classificado tenha um valor $x$, o segundo, $x / 2$, o terceiro, $x / 3$, até o enésimo município, respeitando a proporcionalidade, $x / n$. Valores de $\mathrm{k}<1$ indicam uma maior ubiquidade entre as cidades. No limite, caso $\mathrm{k}=0$, inexiste hierarquia na variável, ou seja, os valores para cada município são suficientemente semelhantes para não indicar a presença de uma hierarquia.

Sob outra perspectiva, substituindo-se a população pelo número de patentes depositadas na regressão, permite-se uma comparação das distribuições, obtendo-se, assim, a classificação do domínio das cidades quanto à sua produção de patentes. O coeficiente da distribuição de patentes é geralmente ainda mais acentuado que o da população, pois as patentes são mais hierarquizadas que a população.

Pode-se estimar a mesma regra de classificação para as patentes. Tem-se, então:

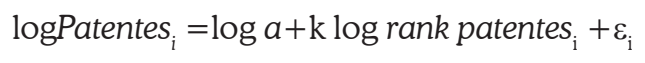

em que log patentes é o logaritmo natural das patentes de uma dada cidade em determinado período e log rank patentes é o logaritmo natural da classificação 
(ranking) de uma dada cidade em determinado período de acordo com o seu total de patentes, sendo k o parâmetro estimado. ${ }^{1}$

De outro modo, buscando-se comprovar as evidências de que a urbanização está, ao menos, correlacionada com externalidades que beneficiam a inovação, através das interações entre as pessoas, da troca de conhecimento, de corporações focadas em PEBD, entre outros, promovendo, assim, progresso tecnológico, foi estimada uma equação na qual se pôde analisar a relação entre o número de pessoas em uma determinada cidade e seus efeitos sobre a produção de patentes. Assim, será estimada a seguinte equação:

$$
\log _{\text {Patentes }_{\mathrm{i}}}=\alpha+\beta \log \text { População }_{\mathrm{i}}+\varepsilon_{\mathrm{i}}
$$

em que log patentes será o log da média de patentes municipais ${ }^{2}$ entre os anos 2006-2009 e log população será a população de 2005 de uma dada cidade.

Diversos modelos lineares sem dependência espacial foram testados a partir do método dos mínimos quadrados ordinários (MQO). Testes de dependência espacial apontaram a necessidade da utilização de métodos de econometria espacial a partir da violação dos pressupostos do modelo clássico de regressão linear. Identificou-se a presença de dependência espacial, o que indica necessidade de tratamento da heterogeneidade espacial. Anselin (1999) recomenda incorporar dependência espacial ao modelo de regressão linear padrão a partir de dois procedimentos: primeiro, introduz-se um regressor adicional na forma de uma variável dependente defasada espacialmente (SAR), que será o modelo de defasagem espacial apropriado para a avaliação da existência e grau de interação espacial; segundo, incorpora-se a dependência espacial presente nas variáveis explicativas para se captar os transbordamentos originados nas regiões vizinhas.

A partir dos procedimentos implementados, concluiu-se que o modelo que melhor se ajusta econometricamente ao problema de endogeneidade espacial é um modelo de Durbin espacial, estimado a partir do método de mínimos quadrados em dois estágios ponderados espacialmente, que produz coeficientes estimados das variáveis $W_{1} y$ e $W_{1} X \beta$, estruturado da seguinte forma, segundo Almeida (2012):

ou a forma estrutural:

$$
y=\rho W_{1} y+X \beta+\rho W_{1} X \beta+\varepsilon
$$

1 Como muitos municípios não possuem patentes, para se aplicar logaritmos, foi usada a seguinte transformação nos dados de patentes: log[patentes +1 ].

2 Esse trabalho enfoca a capacidade de invenção de municípios brasileiros. Para tal, utiliza informações sobre a residência dos inventores no Brasil. Logo, não são usados dados dos depositantes das patentes, que podem ser multinacionais estrangeiras com endereço no Brasil. Essas empresas depositam patentes no Brasil simplesmente para proteger mercado, sem, necessariamente, usar alguma capacidade de invenção do município em que estão localizadas. Para diferenças entre patentes de residentes e não residentes, consultar Barcelos et al. (2014) e Chiarini, Rapini e Silva (2016). 


$$
y=\rho W_{1} y+X \beta-\lambda W_{1} X \beta+\varepsilon
$$

Logo, aplicando-os ao modelo construído:

LogPatentes $=\rho_{1} \mathrm{~W}_{1}$ LogPatentes $\beta_{1} \operatorname{logPopulação}{ }_{\mathrm{i}}+\beta_{2} \mathrm{INT}_{\mathrm{i}}+\beta_{3} \mathrm{ESC}_{\mathrm{i}}+\beta_{4} \mathrm{DPC}_{\mathrm{i}}+\beta_{5} \mathrm{M}_{\mathrm{i}}$ $+\rho_{2} \mathrm{~W}_{1} \operatorname{logPopulação} \mathrm{i}_{\mathrm{i}} \rho_{3} \mathrm{~W}_{1} \mathrm{INT}_{\mathrm{i}}+\rho_{4} \mathrm{~W}_{1} \mathrm{ESC}_{\mathrm{i}}+\varepsilon$

em que a definição das variáveis é apresentada no Quadro 1.

Quadro 1 - Descrição das variáveis construídas para utilização

na estimação do modelo

\begin{tabular}{|c|c|}
\hline Variável & Descrição \\
\hline Log patentes & $\begin{array}{l}\text { Logaritmo natural da média de patentes municipais consi- } \\
\text { derando-se o endereço de residência do inventor no Brasil. } \\
\text { São testados dois períodos: } 2001 \text { a } 2004 \text { e } 2006 \text { a } 2009 \text {. }\end{array}$ \\
\hline Log população & $\begin{array}{l}\text { Logaritmo natural da população do município i, medido em } \\
\text { dois anos alternativamente: } 2000 \text { e } 2005 \text {. }\end{array}$ \\
\hline $\begin{array}{l}\text { Setores intensivos } \\
\text { em tecnologia } \\
\text { (INT) }\end{array}$ & $\begin{array}{l}\text { Proporção de trabalhadores empregados nos setores da } \\
\text { indústria intensivos em tecnologia, medida tanto em } 2000 \\
\text { quanto em 2005, do município i, de acordo com a classifica- } \\
\text { ção da OCDE (2011) e informações constantes no Registro } \\
\text { Anual de Informações Sociais (Rais). }\end{array}$ \\
\hline $\begin{array}{l}\text { Trabalhadores } \\
\text { com curso su- } \\
\text { perior completo } \\
\text { (ESC) }\end{array}$ & $\begin{array}{l}\text { Proporção de trabalhadores com curso superior completo } \\
\text { do município i, medida em } 2000 \text { e em 2005, de acordo com } \\
\text { dados extraídos da Rais. }\end{array}$ \\
\hline $\begin{array}{l}\text { Doutores per } \\
\text { capita (DPC) }\end{array}$ & $\begin{array}{l}\text { Número de doutores per capita, por } 10 \text { mil pessoas, em } \\
\text { áreas de transferência de tecnologia (como ciências exatas } \\
\text { e da terra, ciências agrárias, ciências biológicas e engenha- } \\
\text { rias) para o município i, medido em } 2000 \text { e em } 2005 \text {, obtido } \\
\text { pela Coordenação de Aperfeiçoamento de Pessoal de Nível } \\
\text { Superior (Capes), referente a 1999, e normalizado pela po- } \\
\text { pulação de cada município, conforme Gonçalves (2006). }\end{array}$ \\
\hline $\begin{array}{l}\text { Dummies regio- } \\
\text { nais }(\mathrm{M}) \text { e dummy } \\
\text { para controlar } \\
\text { número de zeros }\end{array}$ & $\begin{array}{l}\text { Vetor de variáveis dummies, constituído por quatro variá- } \\
\text { veis, para representar diferenças macrorregionais de sistema } \\
\text { de inovação e uma dummy para controlar número de zeros } \\
\text { na amostra. }\end{array}$ \\
\hline WlogPatentes & $\begin{array}{l}\text { Variável de defasagem espacial construída a partir da variá- } \\
\text { vel dependente de acordo com uma matriz de pesos espa- } \\
\text { ciais definida. }\end{array}$ \\
\hline$\rho$ & Coeficiente autorregressivo espacial. \\
\hline
\end{tabular}

Fonte: Elaboração própria. 
Como a variável dependente é o nível de patentes da cidade, é necessário inserir variáveis que potencialmente influenciam a produção tecnológica. Segundo a literatura, a produção tecnológica depende da existência de centros urbanos, de setores mais avançados tecnologicamente, de capital humano e pesquisadores universitários, assim como características institucionais próprias de cada região (CARLINO, 2001; FELDMAN; FLORIDA, 1994; FISCHER; VARGA, 2003). Logo, busca-se, a partir das variáveis (ver Quadro 1) e das equações de regressão, identificar as influências do tamanho urbano, da proporção de empregos nos setores de alta e média alta tecnologia, da escolaridade da população adulta, do número de doutores per capita, além das dummies macrorregionais na produção de patentes no período de 2000 a 2009.

\section{Atividade Tecnológica e Cidades no Brasil}

Este artigo mede atividade tecnológica do município com dados de depósitos de patentes (INPI, 2012). A base de dados utilizada é a Base de Dados Estatísticos de Propriedade Intelectual (BADEPI), fornecida pelo Instituto Nacional de Propriedade Intelectual (INPI), referente ao período de 2000 a 2009. A BADEPI reúne as informações referentes às patentes de inventores de municípios brasileiros por pessoa física e jurídica e permite a localização do inventor, razão pela qual as patentes são usadas como proxy de invenção das cidades. Para informações sobre população e trabalhadores, foram coletados dados do Instituto Brasileiro de Geografia e Estatística - IBGE (IBGE, 2016) e da Relação Anual de Informações Sociais (RAIS) do Ministério do Trabalho e Emprego (BRASIL, 2016).

Como visto na revisão de literatura previamente apresentada neste trabalho, em que vários autores defendem a ideia de que o porte das cidades pode estar positivamente relacionado com o seu potencial inovador, as variáveis utilizadas no trabalho foram expostas por categorias de tamanho das cidades, como demonstrado na Tabela 1. 


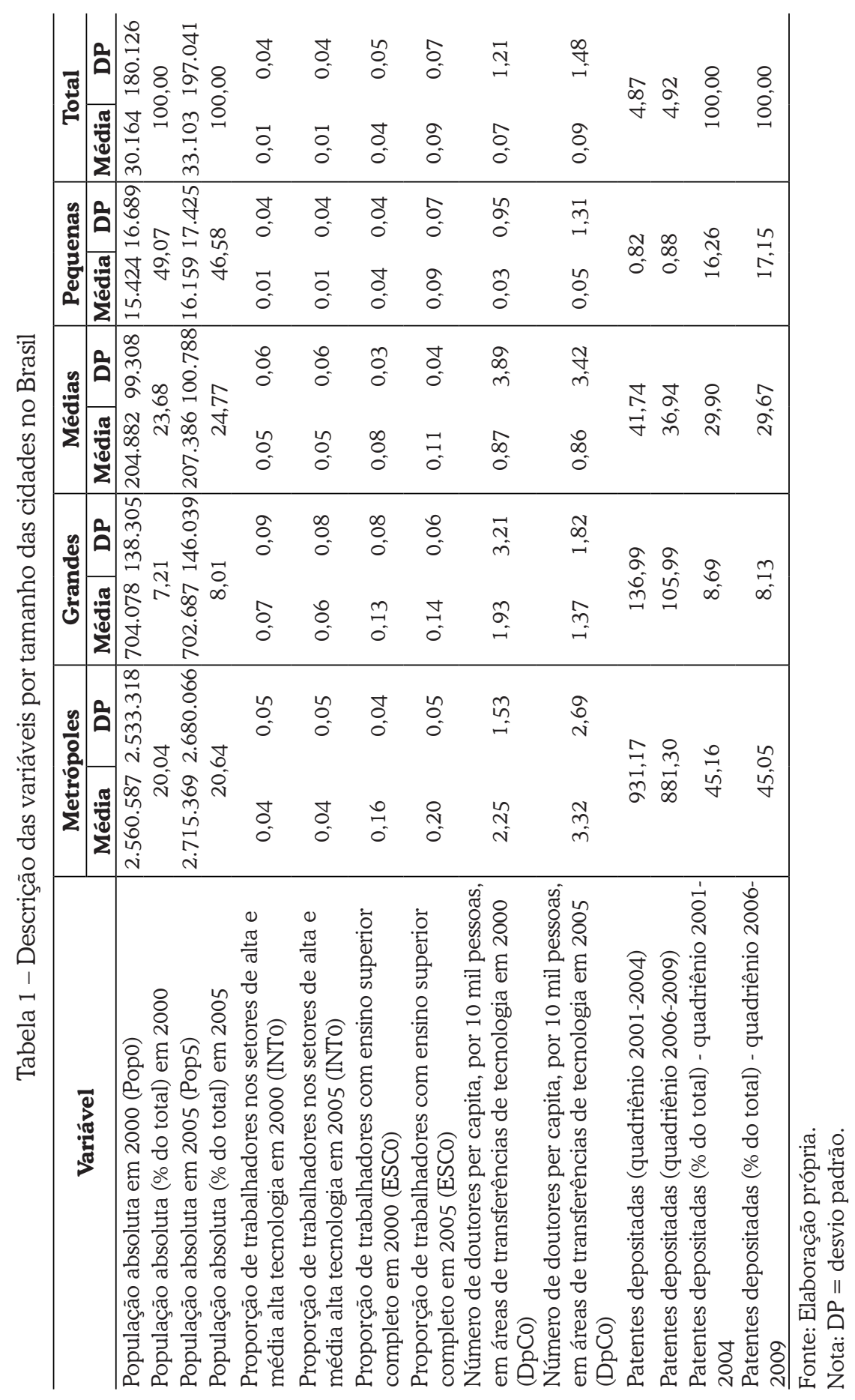


O critério de classificação de municípios como metrópoles, cidades grandes, médias e pequenas foi elaborado, para fins de construção da Tabela 1, tendo como base as seguintes categorias:
a) metrópoles: igual ou maior que 1 milhão de pessoas;
b) cidades grandes: de 500 mil até 999.999 mil pessoas;
c) cidades médias: de 100 mil até 499.999 mil pessoas;
d) cidades pequenas: 0 a 99.999 mil pessoas.

Na Tabela 1 é observado, por meio das variáveis população absoluta em 2000 (Pop0) e população absoluta em 2005 (Pop5) que, em geral, houve um aumento da média da população de um período para o outro. Constata-se que a participação no total da população das cidades classificadas como metrópoles grandes e médias aumentou no período 2000-2005, em detrimento das cidades pequenas, cuja participação caiu de $49 \%$ para $46 \%$.

A concentração de empregados em setores de maior intensidade tecnológica (média-alta e alta tecnologia, nos critérios da OECD (2011) é maior nas cidades de maior porte. Em média, a proporção das pessoas empregadas nesses setores (variável INT0) alcança $25 \%$ nas cidades consideradas metrópoles ou $27 \%$ nas cidades grandes, o que contrasta com a média da proporção das cidades pequenas que se situa apenas em $4 \%$. Além disso, houve tendência de maior crescimento dessa variável nas metrópoles no ano de 2005.

A proporção de trabalhadores com curso superior completo em 2000 (ESC0) também é muito elevada nas maiores cidades. Em média, 16\% dos trabalhadores das metrópoles possuem curso superior completo, contra apenas $4 \%$ de média do indicador nas cidades pequenas. Por outro lado, comparando-se 2005 com 2000, nota-se uma evolução positiva das médias de escolaridade superior em todas as categorias de municípios.

De forma adicional, avaliando-se a variável doutores por 10 mil pessoas (DPC), em áreas passíveis de geração e transferência de tecnologia em 2000 (DPC0), a qual inclui as áreas tecnológicas das ciências exatas e da terra, ciências agrárias, ciências biológicas e engenharias, verifica-se que as metrópoles possuem em média 2,25 doutores por 10 mil pessoas. As cidades grandes possuíam em média 1,93 doutores per capita, enquanto as cidades médias 0,87 e as pequenas 0,03. Em 2005, nota-se que houve um aumento do número de doutores per capita, exceto nas cidades grandes e médias onde houve uma redução para 1,37 e 0,86 doutores per capita, respectivamente.

Sob a perspectiva da análise de patentes, nota-se que a média de patentes depositadas nos períodos 2001-2004 e 2006-2009 foi significativamente maior nas metrópoles, apresentando, em média, 931,17 patentes depositadas em 2001-2004 e 881,30 patentes em 2006-2009. Em comparação com as outras categorias, as cidades grandes seguem na frente com 136,99 patentes depositadas em 2001-2004 
e 105,99 em 2006-2009, seguidas pelas cidades médias com 41,74 patentes em média em 2001-2004 e 36,94 em 2006-2009.

Por fim, verifica-se que as metrópoles possuem um percentual de $45 \%$ do total de patentes depositadas em ambos os períodos 2001-2004 e 2006-2009, o que é consideravelmente maior do que as outras categorias. As cidades médias possuem uma parcela também expressiva desse percentual de $29 \%$ em ambos os períodos. Já as cidades pequenas possuem um percentual de 16\% em 2001-2004 e $17 \%$ em 2006-2009.

Corroborando esse resultado, por meio da Figura 1 pode-se identificar as cidades com maior potencial tecnológico quanto à geração de patentes. Vê-se que não houve mudança substancial quanto à produção de patentes, permanecendo nos rankings os mesmos municípios, havendo poucas variações de posições de um período para o outro, como os municípios de Vitória (ES), São Caetano do Sul (SP), Osasco (SP), Novo Hamburgo (RS) e Marília (SP), que cederam lugar aos municípios de Jundiaí (SP), Piracicaba (SP), Chapecó (SC), Sorocaba (SP) e Manaus (AM). Há certa estabilidade na parte superior da Figura 1. 
Figura 1 - Ranking dos 30 maiores municípios patenteadores construído a partir da soma de patentes depositadas nos municípios

nos períodos 2001-2004 e 2006-2009

\begin{tabular}{|c|c|c|c|c|c|c|}
\hline & \multicolumn{3}{|c|}{$2001-2004$} & \multicolumn{3}{|c|}{$2006-2009$} \\
\hline Ordem & UF & Munic ípio & Patentes & UF & Município & Patentes \\
\hline 1 & SP & SãoPaub & 592426 & $\mathrm{SP}$ & São Paulo & 5389,88 \\
\hline 2 & $\mathrm{RJ}$ & Rio de Janeiro & 1472,16 & $\mathrm{RJ}$ & Rio de Janeiro & 1383,04 \\
\hline 3 & PR & Curitiba & 1219,85 & PR & Curitiba & 1291,03 \\
\hline 4 & $\mathrm{MG}$ & Belo Horizonte & 1089,74 & $\mathrm{MG}$ & Belo Horizonte & 1068,68 \\
\hline 5 & RS & Porto Alegre & 756,78 & $\mathrm{RS}$ & Porto Alegre & 741,704 \\
\hline 6 & SP & Campinas & 700,80 & $\mathrm{SP}$ & Campinas & 704,365 \\
\hline 7 & $\mathrm{SC}$ & Joinville & 463,82 & $\mathrm{SC}$ & Joinville & 455,611 \\
\hline 8 & RS & Caxias do Sul & 436,13 & $\mathrm{DF}$ & Brasilia & 438,714 \\
\hline 9 & $\mathrm{DF}$ & Brasilia & 419,80 & $\mathrm{RS}$ & Caxias do Sul & 403,743 \\
\hline 10 & SP & São Bernardo do Campo & 406,28 & $\mathrm{C}$ & Florianópolis & 331,568 \\
\hline 11 & $\mathrm{SC}$ & Florianópolis & 349,16 & $\mathrm{SP}$ & São Bemardo do Campo & 281,97 \\
\hline 12 & GO & Goùt nia & 261,79 & $\mathrm{SP}$ & São José dos Campos & 276,499 \\
\hline 13 & SP & Santo André & 247,40 & $\mathrm{GO}$ & Goiânia & 253,947 \\
\hline 14 & $\mathrm{CE}$ & Fortaleza & 244,31 & $\mathrm{CE}$ & Fortaleza & 253,394 \\
\hline 15 & SP & São José dos Campos & 218,15 & SP & Guaruthos & 242,168 \\
\hline 16 & SP & Guarulhos & 214,28 & $\mathrm{~A}$ & Salvador & 239,921 \\
\hline 17 & $\mathrm{PE}$ & Recife & 204,34 & SP & São Carlos & 232,525 \\
\hline 18 & $\mathrm{SC}$ & Bhumenau & 200,62 & $P$ & Ribeirão Pre to & 227,858 \\
\hline 19 & BA & Salvador & 198,55 & & Maringá & 224,379 \\
\hline 20 & SP & Ribeirão Preto & 193,70 & & Santo André & 219,687 \\
\hline 21 & PR & Maringá & 184,35 & $\mathrm{SC}$ & Blumenau & 204,55 \\
\hline 22 & PR & Londrina & 174,92 & $\mathrm{SP}$ & Jundiai & 184,725 \\
\hline 23 & RS & Novo Hamburgo & 168,63 & $\mathrm{R}$ & Londrina & 174,148 \\
\hline 24 & SP & São José do Rio Preto & 161,72 & $\mathrm{PE}$ & Recife & 170,365 \\
\hline 25 & RS & Passo Fundo & 155,17 & ES & Vitória & 157,896 \\
\hline 26 & SP & São Carlos & 154,33 & $P$ & Piracicaba & 149,813 \\
\hline 27 & SP & Piracicaba & 154,12 & $\mathrm{SC}$ & Chapecó & 146,667 \\
\hline 28 & SP & Barueri & 152,51 & RJ & Niterói & 144,363 \\
\hline 29 & $\mathrm{RJ}$ & Niteröi & 152,21 & SP & Barueri & 141,437 \\
\hline 30 & SP & Jundiai & 151.77 & SP & Bauru & 137576 \\
\hline
\end{tabular}

Fonte: Elaboração própria.

A estimação do coeficiente de Zipf evidencia tais efeitos de hierarquia urbana para as distribuições de população e patentes em dois períodos distintos: a população de 2000 e a média de patentes para o período 2001-2004 e a população de 2005 e a média de patentes de 2006 a 2009, de acordo com o Gráfico 1. 
Grafico 1 - Gráfico log x log da distribuição hierárquica dos municípios brasileiros para a população e patentes em diferentes períodos
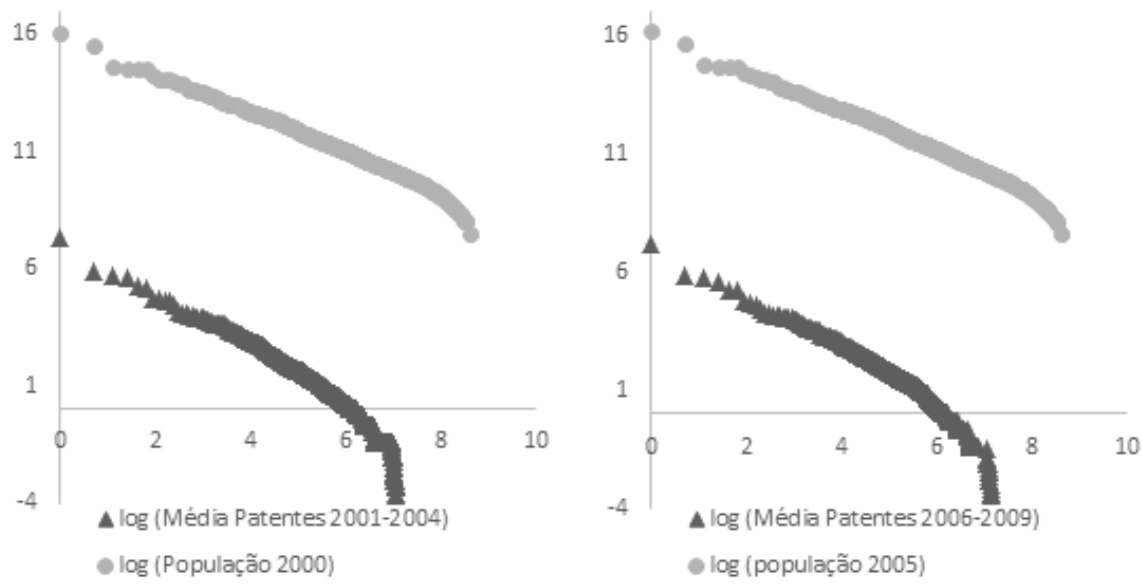

Fonte: Elaboração própria com base em dados da Badepi/Inpi (versão 1.0) (INPI, 2012) e dados populacionais fornecidos pelo IBGE (2016).

Nota: O eixo horizontal é a classificação da região com base no critério de tamanho populacional ou tamanho de patentes (quanto menor a classificação, maior a frequência da região).

Observa-se, por meio do Gráfico 1, que, em ambos os períodos, as patentes são mais concentradas que a população, tomando a inclinação das retas como critério. Percebe-se também que a distribuição da população apresenta característica mais linear que a distribuição das patentes. Um grande número de regiões com baixo valor de patentes e menos de uma patente ${ }^{3}$ também é evidenciado na figura. Os resultados do ajuste de uma regressão linear simples são apresentados na Tabela 2.

3 Patentes cujos inventores se localizam em diferentes municípios foram atribuídas proporcionalmente aos diferentes municípios. Por exemplo, um processo de patente com dois inventores de municípios $X$ e $Y$ faz com que estes tenham, respectivamente, 0,5 patentes cada um. 
Tabela 2 - Resultados dos coeficientes de Zipf para as regressões de população e patentes nos períodos selecionados

\begin{tabular}{|c|c|c|c|c|}
\hline Variável & $\begin{array}{c}\text { (1) } \\
\log (\text { popula- } \\
\text { ção 2000) }\end{array}$ & $\begin{array}{c}\text { (2) } \\
\text { log (média } \\
\text { patentes } \\
2001-2004 \text { ) }\end{array}$ & $\begin{array}{c}\text { (3) } \\
\log (p o p u- \\
\text { lação 2005) }\end{array}$ & $\begin{array}{c}\text { (4) } \\
\text { log (média } \\
\text { patentes } \\
2006-2009 \text { ) }\end{array}$ \\
\hline $\begin{array}{l}\text { log (rank população } \\
\text { 2000) }\end{array}$ & $\begin{array}{c}-1,09 * * * \\
(0,00)\end{array}$ & & & \\
\hline $\begin{array}{l}\text { log (rank média } \\
\text { patentes 2001-2004) }\end{array}$ & & $\begin{array}{c}-1,52 * * * \\
(0,01)\end{array}$ & & \\
\hline $\begin{array}{l}\log \text { (rank população } \\
\text { 2005) }\end{array}$ & & & $\begin{array}{c}-1,12 * * * \\
(0,00)\end{array}$ & \\
\hline $\begin{array}{l}\text { log (rank média paten- } \\
\text { tes 2006-2009) }\end{array}$ & & & & $\begin{array}{c}-1,53 * * * \\
(0,01)\end{array}$ \\
\hline Constante & $\begin{array}{c}17,62^{* * *} \\
(0,03)\end{array}$ & $\begin{array}{c}9,08^{* * *} \\
(0,07)\end{array}$ & $\begin{array}{c}17,90 * * * \\
(0,03)\end{array}$ & $\begin{array}{c}9,23 * * * \\
(0,07)\end{array}$ \\
\hline Observações & 5.507 & 1.133 & 5.564 & 1.246 \\
\hline $\mathrm{R}^{2}$ & 0,94 & 0,94 & 0,94 & 0,94 \\
\hline
\end{tabular}

Fonte: Elaboração própria com auxílio do software Stata 13.

Nota: Erro padrão entre parênteses; nível de significância: * $\mathrm{p}<0,1$; ** $\mathrm{p}<0,05$; ** $\mathrm{p}<$ 0,01 .

Os coeficientes de hierarquia populacional e de patentes em ambos os períodos indicam hierarquização mais que proporcional pelos maiores centros urbanos. Os resultados estão de acordo com a literatura, uma vez que as patentes apresentam maior coeficiente de Zipf que a população, sendo mais desigualmente distribuídas que a última. Além disso, o $R^{2}$ indica que há um ajuste linear mais eficiente na regressão de patentes, o que se deve à presença de não linearidade na cauda inferior da distribuição populacional, que prejudica o ajuste.

A Tabela 3 apresenta os resultados da estimação por MQO da equação 5. Obteve-se como resultado um coeficiente positivo e significativo de 0,30 e 0,31 para 2001-2004 e 2006-2009, respectivamente, indicando que o tamanho urbano influencia positivamente a produção de patentes. Um aumento de $1 \%$ da população aumenta em $0,3 \%$ o nível médio de patentes da cidade. 
Tabela 3 - Resultados da estimação por MQO

(variável dependente: log do nível médio de patentes no período)

\begin{tabular}{ccc}
\hline & Log patentes 2001-2004 & Log patentes 2006-2009 \\
\hline Constante & $-2,63^{* * *}$ & $-2,68^{* * *}$ \\
Log população 2000 & $(0,12)$ & $(0,12)$ \\
& $0,30^{* * *}$ & \\
Log população 2005 & $(0,01)$ & $0,31 * * *$ \\
& & $(0,01)$ \\
\hline Observações & & 5.507 \\
$\mathrm{R}^{2}$ ajustado & 5.507 & 0,37 \\
\hline
\end{tabular}

Fonte: Elaboração própria a partir do software Stata 13.

Nota: Erro padrão robusto entre parênteses; nível de significância: * $p<0,1$; ** $p<0,05$; $* * * \mathrm{p}<0,01$.

Identificada a presença de hierarquias urbanas, busca-se evidenciar os efeitos dos determinantes das invenções sob influência das escalas urbanas. Para isso, adiciona-se um conjunto de controles relacionados aos determinantes da atividade tecnológica. São apresentados na Tabela 3 os resultados para o período (i) 2001-2004 e (ii) 2006-2009, estimadas pelo método MQO com a adição de variáveis de controle. Na primeira e terceira colunas, não foram acrescentadas as variáveis dummies para as macrorregiões, enquanto que, na segunda e quarta, os resultados estão acrescidos dessas variáveis. Em geral, as variáveis dependentes dos trabalhos que usam econometria são medidas em termos relativos, como patentes per capita. Nesse caso, como se pretende adicionar controles urbanos para se testar a robustez da relação entre nível populacional e nível de patentes, usou-se o número de patentes absoluto (na média do período) como variável dependente. Tal procedimento também é realizado por Ó hUallacháin (1999) nos seus testes econométricos entre tamanho urbano e nível de patentes, embora o autor não realize o controle para dependência espacial entre as observações. As regressões estimadas ao nível de município implicam em número excessivo de zeros na variável dependente. Para controlar isso, incluiu-se uma variável dummy que assumiu o valor um caso o município tivesse patentes e zero se não possuísse. O valor da dummy é significativo e sua inclusão não retira significâncias das outras variáveis.

A Tabela 4 está dividida em três partes. Na primeira, são apresentados os coeficientes e suas significâncias estatísticas. Na segunda, os testes de normalidade dos resíduos Jarque-Bera, de heterocedasticidade, Koenker-Basset, de multicolinearidade, a estatística $R^{2}$ e a estatística $F$. Na terceira parte, estão os testes de diagnóstico para autocorrelação espacial. 
Tabela 4 - Resultados do modelo estimado por MQO

(variável dependente: log patentes)

\begin{tabular}{|c|c|c|c|c|}
\hline \multirow{2}{*}{ Variável } & \multicolumn{2}{|c|}{ 2001-2004 } & \multicolumn{2}{|c|}{ 2006-2009 } \\
\hline & (1) & (2) & (3) & (4) \\
\hline \multirow{2}{*}{ Constante } & $-1,64 * * *$ & $-1,76 * * *$ & $-1,73 * * *$ & $-1,91 * * *$ \\
\hline & $(0,06)$ & $(0,05)$ & $(0,05)$ & $(0,05)$ \\
\hline \multirow{2}{*}{ Log população (POP) } & $0,18 * * *$ & $0,19 * * *$ & $0,18 * * *$ & $0,21 * * *$ \\
\hline & $(0,12)$ & $(0,01)$ & $(0,01)$ & $(0,01)$ \\
\hline \multirow{2}{*}{$\begin{array}{l}\text { Setores intensivos em } \\
\text { tecnologia (INT) }\end{array}$} & $0,39 * *$ & $0,36 * * *$ & $0,42 * * *$ & $0,37 * *$ \\
\hline & $(0,04)$ & $(0,04)$ & $(0,03)$ & $(0,03)$ \\
\hline \multirow{2}{*}{$\begin{array}{l}\text { Trabalhadores com curso } \\
\text { superior completo (ESC) }\end{array}$} & $1,28 * * *$ & $0,99 * * *$ & $0,52 * * *$ & $0,53 * * *$ \\
\hline & $(0,12)$ & $(0,12)$ & $(0,08)$ & $(0,07)$ \\
\hline \multirow{2}{*}{ Doutores per capita (DPC) } & $0,06 * * *$ & $0,06 * *$ & $0,05^{* *}$ & $0,050 * *$ \\
\hline & $(0,00)$ & $(0,00)$ & $(0,00)$ & $(0,00)$ \\
\hline \multirow{2}{*}{$\begin{array}{l}\text { Dummy patente (valor } 1 \text { se o } \\
\text { município possui patente) }\end{array}$} & $0,55 * *$ & $0,49 * * *$ & $0,57 * *$ & $0,47 * * *$ \\
\hline & $(0,01)$ & $(0,02)$ & $(0,01)$ & $(0,02)$ \\
\hline \multirow{2}{*}{ Dummy região norte $(\mathrm{N})$} & - & $-0,07^{* * *}$ & - & $-0,11 * * *$ \\
\hline & & $(0,02)$ & & $(0,02)$ \\
\hline \multirow{2}{*}{ Dummy região nordeste (NE) } & - & $-0,11 * * *$ & - & $-0,14^{* * *}$ \\
\hline & & $(0,01)$ & & $(0,01)$ \\
\hline \multirow{2}{*}{ Dummy região sul (S) } & - & $0,04 * * *$ & - & $0,06 * * *$ \\
\hline & & $(0,01)$ & & $(0,02)$ \\
\hline \multirow{2}{*}{$\begin{array}{l}\text { Dummy região centro-oeste } \\
\text { (CO) }\end{array}$} & - & $-0,02$ & - & $-0,04^{*}$ \\
\hline & & $(0,02)$ & & $(0,02)$ \\
\hline $\mathrm{R}^{2}$ ajustado & 0,55 & 0,56 & 0,55 & 0,57 \\
\hline $\begin{array}{l}\text { Condição de multicolineari- } \\
\text { dade }\end{array}$ & 24,3 & 27,8 & 23,9 & 27,7 \\
\hline AIC & 4748,1 & 4649,1 & 5147,9 & 4972,3 \\
\hline $\mathrm{SC}$ & 4787,8 & 4715,2 & 5187,6 & 5038,4 \\
\hline Estatística de Jarque-Bera & $163711,5^{* * *}$ & $156484,6 * * *$ & $15614,1 * * *$ & $15643,4 * * *$ \\
\hline Teste de Koenker-Basset & $1268,2^{* * *}$ & $1303,4 * * *$ & $1308,5^{* * *}$ & $15643,4^{* * *}$ \\
\hline Estatística F & $1367,8 * *$ & $786,2^{* * *}$ & $1357,5^{* * *}$ & $1378,1 * * *$ \\
\hline \multicolumn{5}{|c|}{ Teste de dependência espacial (matriz de pesos espacial: queen) } \\
\hline Lagrange multiplier (lag) & $493,1 * *$ & $402,3 * *$ & $486,3^{* * *}$ & $344,7 * *$ \\
\hline Robust LM (lag) & $99,8 * * *$ & $58,5 * *$ & $108,6 * * *$ & $43,6 * * *$ \\
\hline
\end{tabular}


conclusão.

\begin{tabular}{lcccc}
\hline \multirow{2}{*}{ Variável } & \multicolumn{2}{c}{ 2001-2004 } & \multicolumn{2}{c}{ 2006-2009 } \\
\cline { 2 - 5 } & (1) & (2) & (3) & (4) \\
\hline Lagrange multiplier (error) & $487,3^{* * *}$ & $442,1^{* * *}$ & $454,1^{* * *}$ & $397,2^{* * *}$ \\
Robust LM (error) & $94,1^{* * *}$ & $98,4^{* * *}$ & $76,3^{* * *}$ & $96,1^{* * *}$ \\
Lagrange multiplier (SARMA) & $587,2^{* * *}$ & $500,7^{* * *}$ & $562,7^{* * *}$ & $440,8^{* * *}$ \\
\hline
\end{tabular}

Fonte: Elaboração própria por meio do pacote econométrico do GeoDaSpace.

Nota: Erro-padrão entre parênteses; nível de significância: * $\mathrm{p}<0,1$; ** $\mathrm{p}<0,05$; ** $\mathrm{p}<$ 0,01 .

A relação entre população e patentes é positiva e significativa, mesmo após adição de controles urbanos e regionais. O coeficiente da variável população é menor do que aquele estimado através da equação (5) - Tabela 3, que não inclui controles adicionais. Porém, nas regressões acrescidas das dummies, os coeficientes se elevaram, com coeficientes negativos para as dummies centro-oeste, norte e nordeste e positivos para a região sul. O resultado reforça a importância da base industrial e tecnológica dos municípios do sul do Brasil. Estatísticas descritivas como patentes por 1 milhão de pessoas revelam que, enquanto o estado de São Paulo apresentou queda no patenteamento per capita de 87,22 (média 2001-2004) para 75,48 (média 2006-2009), os estados do sul aumentaram ou apresentaram pequena redução no mesmo período. O estado do Paraná aumentou sua média de 62,96 para 63,45 e os estados de Santa Catarina e Rio Grande do Sul apresentaram menor redução, respectivamente, de 97,90 e 63,73 para 97,44 e 60,41.

De modo geral, a inclusão das dummies aumentou o poder de explicação dos modelos, comparando-se os indicadores $R^{2}$ e as estatísticas AIC e SC na segunda parte da Tabela 4. Isso demonstra que a adição de controles na regressão não afetou a significância da relação positiva encontrada na Tabela 3, entre o tamanho populacional e o nível de patentes. Esse resultado indica que não só o tamanho do município, mas também sua localização, explicam a magnitude de suas invenções.

Nas outras variáveis de controle, pode-se observar que todos os coeficientes apresentam o sinal esperado pela literatura. A proporção de pessoal empregado nos setores de alta e média alta tecnologia (INT) influencia positivamente a atividade inovadora. Além disso, as variáveis proporção de trabalhadores com curso superior completo (ESC) e número de doutores em áreas com potencial de geração e transferência tecnológica (DPC) apresentaram resultado positivo e significativo, em todas as regressões, indicando a importância da qualificação das pessoas e da capacidade de pesquisa e desenvolvimento universitário, respectivamente, para o desenvolvimento de atividades tecnológicas, como mostrado na literatura do presente artigo. O uso dessas variáveis sugere que a atividade inventiva está asso- 
ciada com outros fatores além do tamanho absoluto da população, mas também influenciados pela escala urbana (SIMMIE, 2001; FELDMAN; FLORIDA, 1994).

Vê-se que não há problemas relacionados à multicolinearidade nas variáveis nos dois modelos. Além disso, por meio da estatística de Jarque-Bera, pode-se rejeitar a hipótese de normalidade dos resíduos, e os testes de Koenker-Basset apontam que os resíduos não são homocedásticos. Na última parte da Tabela 4, estão expostos os testes de dependência espacial, o multiplicador de Lagrange para a defasagem espacial da variável dependente, LM-LAG, e para o erro autorregressivo, LM-ERR, para a matriz de pesos espaciais que apresentou o maior I de Moran significativo, a matriz queen.

Os testes para identificação de dependência espacial indicam que os resíduos da regressão não são homogeneamente distribuídos, indicando a necessidade do controle da autocorrelação espacial nos modelos estimados. Visto que, os testes LM_ERR (robusto) e LM-LAG (robusto) não foram conclusivos quanto à dependência espacial no erro ou na forma de uma defasagem espacial da variável dependente, optou-se por controlar a autocorrelação espacial por meio da defasagem espacial da variável dependente e das variáveis explicativas, log população, trabalhadores em setores intensivos em tecnologia e trabalhadores com ensino superior. ${ }^{4}$

A Tabela 5 apresenta um último esforço de estimação tentando corrigir os problemas de não normalidade dos resíduos e de dependência espacial dos modelos, ambos constatados na Tabela 4. Assim, foi estimado o modelo de Durbin espacial por meio do método de mínimos quadrados em dois estágios, que combina os efeitos de interação endógena entre as defasagens das variáveis dependente e explicativas.

$4 \quad$ Foram testados modelos com defasagem espacial do termo de erro, contudo, os resultados estimados não se apresentaram significativos para a correção da dependência espacial nas estimações. 
Tabela 5 - Resultados do modelo Durbin espacial (SDM) estimado por mínimos quadrados em dois estágios (variável dependente: log patentes)

\begin{tabular}{|c|c|c|c|c|}
\hline \multirow[t]{2}{*}{ Variável } & \multicolumn{2}{|c|}{ 2001-2004 } & \multicolumn{2}{|c|}{ 2006-2009 } \\
\hline & (1) & (2) & (3) & (4) \\
\hline \multirow[t]{2}{*}{ Constante } & $-0,70 * *$ & $-0,83 * * *$ & $-0,84 * * *$ & $-1,03 * * *$ \\
\hline & $(0,15)$ & $(0,16)$ & $(0,13)$ & $(0,14)$ \\
\hline \multirow[t]{2}{*}{ Log população (POP) } & $0,20 * * *$ & $0,20 * * *$ & $0,21 * *$ & $0,21 * * *$ \\
\hline & $(0,01)$ & $(0,01)$ & $(0,01)$ & $(0,01)$ \\
\hline \multirow[t]{2}{*}{ Setores intensivos em tecnologia (INT) } & $0,19 * * *$ & $0,20 * * *$ & $0,28 * * *$ & $0,28 * * *$ \\
\hline & $(0,05)$ & $(0,05)$ & $(0,04)$ & $(0,04)$ \\
\hline \multirow[t]{2}{*}{$\begin{array}{l}\text { Trabalhadores com curso superior com- } \\
\text { pleto (ESC) }\end{array}$} & $1,12 * * *$ & $1,11 * *$ & $0,53 * * *$ & $0,54 * * *$ \\
\hline & $(0,25)$ & $(0,25)$ & $(0,09)$ & $(0,09)$ \\
\hline \multirow[t]{2}{*}{ Doutores per capita (DPC) } & $0,06 * * *$ & $0,06 * * *$ & $0,05 * * *$ & $0,05 * *$ \\
\hline & $(0,02)$ & $(0,02)$ & $(0,01)$ & $(0,01)$ \\
\hline \multirow[t]{2}{*}{$\begin{array}{l}\text { Dummy patente (valor } 1 \text { se o município } \\
\text { possui patente) }\end{array}$} & $0,44 * * *$ & $0,43 * *$ & $0,44 * * *$ & $0,42 * * *$ \\
\hline & $(0,02)$ & $(0,02)$ & $(0,02)$ & $(0,02)$ \\
\hline \multirow[t]{2}{*}{ Dummy região norte $(\mathrm{N})$} & - & 0,01 & - & $-0,01$ \\
\hline & & $(0,02)$ & & $(0,02)$ \\
\hline \multirow[t]{2}{*}{ Dummy região nordeste (NE) } & - & $-0,01$ & - & $-0,03 * *$ \\
\hline & & $(0,01)$ & & $(0,01)$ \\
\hline \multirow[t]{2}{*}{ Dummy região sul (S) } & - & $0,05 * *$ & - & $0,05 * *$ \\
\hline & & $(0,02)$ & & $(0,02)$ \\
\hline \multirow[t]{2}{*}{ Dummy região centro-oeste (CO) } & - & 0,02 & - & 0,02 \\
\hline & & $(0,02)$ & & $(0,02)$ \\
\hline \multicolumn{5}{|l|}{ Defasagens espaciais } \\
\hline \multirow[t]{2}{*}{ W log patentes (W_PAT) } & $0,26 * * *$ & $0,240 * * *$ & $0,249 * *$ & $0,226 * *$ \\
\hline & $(0,03)$ & $(0,03)$ & $(0,03)$ & $(0,03)$ \\
\hline \multirow[t]{2}{*}{ W log população (W_POP) } & $-0,12 * * *$ & $-0,11 * * *$ & $-0,12 * * *$ & $-0,11 * * *$ \\
\hline & $(0,02)$ & $(0,02)$ & $(0,02)$ & $(0,02)$ \\
\hline \multirow[t]{2}{*}{$\begin{array}{l}\text { W setores intensivos em tecnologia } \\
\text { (W_INT) }\end{array}$} & $0,83 * * *$ & $0,89 * *$ & $0,64 * * *$ & $0,61 * * *$ \\
\hline & $(0,17)$ & $(0,17)$ & $(0,13)$ & $(0,13)$ \\
\hline \multirow[t]{2}{*}{$\begin{array}{l}\text { W trabalhadores com curso superior } \\
\text { completo (W_ESC) }\end{array}$} & $-1,02 * * *$ & $-1,23 * * *$ & $-0,33 * *$ & $-0,28 *$ \\
\hline & $(0,31)$ & $(0,31)$ & $(0,15)$ & $(0,16)$ \\
\hline
\end{tabular}




\begin{tabular}{|c|c|c|c|c|}
\hline \multirow[t]{2}{*}{ Variável } & \multicolumn{2}{|c|}{ 2001-2004 } & \multicolumn{2}{|c|}{ 2006-2009 } \\
\hline & (1) & (2) & (3) & (4) \\
\hline \multirow[t]{2}{*}{ W doutores per capita (W_DPC) } & $-0,04^{*}$ & $-0,03$ & $-0,01$ & $-0,01$ \\
\hline & $(0,02)$ & $(0,02)$ & $(0,01)$ & $(0,01)$ \\
\hline Pseudo $\mathrm{R}^{2}$ espacial & 0,58 & 0,58 & 0,58 & 0,58 \\
\hline Teste Anselin-Kelejian & $\begin{array}{l}1,79 \\
\text { Prob.: } \\
0,18\end{array}$ & $\begin{array}{l}0,32 \\
\text { Prob.: } \\
0,57\end{array}$ & $\begin{array}{l}1,31 \\
\text { Prob.: } \\
0,25\end{array}$ & $\begin{array}{l}0,04 \\
\text { Prob.: } \\
0,84\end{array}$ \\
\hline
\end{tabular}

Fonte: Elaboração própria por meio do pacote econométrico do GeoDaSpace.

Nota: Erro padrão robusto entre parênteses; nível de significância: * $\mathrm{p}<0,1 ;{ }^{* *} \mathrm{p}<0,05$; $* * * \mathrm{p}<0,01$.

Na Tabela 5, o efeito da escala urbana é identificado por meio da variável população (POP), e seus resultados apontam que a correlação entre ambas é positiva e altamente significativa, o que reforça os resultados interpretados a partir da lei de Zipf. Os demais resultados seguem a Tabela 4 e podem ser interpretados de forma similar.

A variável dependente espacialmente defasada (W log patentes) tem como finalidade testar a hipótese de transbordamento tecnológico espacial (GONÇALVES; ALMEIDA, 2009). Essa variável apresentou sinal positivo e significativo nas quatro especificações da Tabela 5, sugerindo a existência de efeitos de transbordamentos localizados. Isso quer dizer que os municípios patenteadores formam agrupamentos espaciais em que a atividade tecnológica de um influencia positivamente a do outro.

A defasagem espacial da população municipal (W log população) foi negativa e estaticamente diferente de zero. Esse resultado sugere que há relação negativa entre o nível de patenteamento de uma cidade e o nível médio de população dos seus vizinhos, indicando predomínio do nível de patentes em cidades mais populosas. A defasagem espacial do nível de intensidade tecnológica (W_INT) sugere que o patenteamento esteja também correlacionado com o nível tecnológico dos vizinhos, enquanto o nível de trabalhadores defasados espacialmente (W_ESC) apresentou sinal negativo, embora estatisticamente não significativo.

A estatística $\mathrm{R}^{2}$ sugere que o modelo espacial apresenta um melhor ajuste do que os resultados dos modelos apresentados na Tabela 4. O teste de Anselin-Kelejian, para análise de dependência espacial, indica que os modelos estimados corrigem o problema de dependência espacial. 


\section{Considerações Finais}

A partir da aplicação da metodologia de hierarquia urbana e econometria espacial nos dados de patentes por municípios brasileiros, é possível alcançar algumas conclusões que reforçam boa parte da evidência encontrada na literatura internacional para o Brasil.

O primeiro ponto é que existe no Brasil uma alta correlação entre a escala urbana e a geração de invenções. Os principais municípios inventores, considerando-se o número absoluto de patentes, são, em geral, os principais centros urbanos, em termos populacionais, e cidades médias com maior potencial industrial do país. Verifica-se que as metrópoles apresentam os percentuais mais elevados de produção de patentes, com $45 \%$ do total de patentes depositadas nos períodos 2001-2004 e 2006-2009. Há também certa estabilidade quanto aos municípios pertencentes ao ranking de cidades com maior potencial tecnológico em ambos os períodos. Os principais municípios são São Paulo (SP), Rio de Janeiro (RJ), Curitiba (PR), Belo Horizonte (MG), Porto Alegre (RS) e Campinas (SP), além de alguns municípios de porte médio e tradição industrial como Joinville (SC).

Reforçando as constatações, a estimação do coeficiente de Zipf evidencia efeitos de hierarquia urbana para as distribuições de população e patentes, indicando hierarquização mais que proporcional pelos maiores centros urbanos. A distribuição populacional brasileira ao nível de municípios apresenta um ajuste mais próximo ao da lei de Zipf ( $\mathrm{k}=1$ ), porém, ainda assim, é uma hierarquia mais que proporcionalmente distribuída, embora a relação não seja tão hierarquizada como no caso das invenções. O mesmo não se observa na distribuição das patentes, que, por serem mais que proporcionalmente concentradas nas grandes metrópoles, são mais hierarquizadas pelos grandes centros urbanos, o que se refletiu no alto coeficiente de Zipf encontrado. A relação positiva e significativa entre patentes e população no modelo estimado indica os benefícios da escala urbana para a atividade inventiva. A adição de controles, como a proporção de pessoal empregado nos setores de alta e média alta tecnologia (INT), a variável proporção de trabalhadores com curso superior completo (ESC) e número de doutores por 10 mil pessoas em áreas de transferência de tecnologia (DPC), não diminuiu a importância da escala urbana sobre o nível de patentes dos municípios no Brasil.

As técnicas de econometria espacial revelam que a variável dependente espacialmente defasada (W log patentes) é uma determinante relevante do nível de patenteamento municipal. Isso sinaliza a existência de transbordamentos de conhecimento tecnológico entre os municípios para os períodos testados. Tais transbordamentos também são provocados pela participação de trabalhadores em atividades de média-alta e alta tecnologia nas cidades próximas. 
Este trabalho apresenta limitações e possibilidades de extensão futuras. Primeiro, deve-se reconhecer que o uso da unidade regional municipal para o caso brasileiro é questionável pela presença dos arranjos populacionais e pela relação de polarização entre municípios. Logo, pode-se investigar as relações aqui testadas sob escalas regionais mais agregadas do que municípios. Segundo, pode-se investigar as relações de hierarquia urbana e atividade tecnológica considerando-se também os dados de patentes de não residentes versus residentes, ou seja, segundo as informações de depositantes e não somente de inventores brasileiros. Por fim, o uso de outras proxies de atividade tecnológica, além de atividade de patenteamento, pode complementar este estudo, validando ou não as relações encontradas.

\section{Referências}

ACS, Z. J.; FITZROY, F. R.; SMITH, I. High technology employment, wages and university RED spillovers: evidence from US cities. Economics of Innovation and New Technology, v. 8, n. 1-2, p. 57-78, 1999.

ALMEIDA, E. Econometria espacial aplicada. Campinas: Editora Alínea, 2012.

ALMEIDA, P.; KOGUT, B. Localization of knowledge and the mobility of engineers in regional networks. Management Science, v. 45, n. 7, p. 905-917, 1999.

ANSELIN, L. Spatial econometrics: methods and models. Dallas: University of Texas, 1999.

ARTHUR, W. B. Competing technologies, increasing returns, and lock-in by historical events. The Economic Journal, v. 99, p. 116-131, 1989.

AUDRETSCH, D. B.; KEILBACH, M. The mobility of economic agents as conduits of knowledge spillovers. In: FORNAHL, D.; ZELLNER, C.; AUDRETSCH, D. B. (Eds.). The role of labour mobility and informal networks for knowledge transfer. [S. 1.]: Springer, 2005. p. 8-25.

BARCELOS, V. et al. The use of intellectual property in Brazil. [S. 1.], 2014. (Economic Research Working Paper, n. 23). Disponível em: < http://www.wipo.int/edocs/pubdocs/en/wipo_pub_ econstat_wp_23.pdf>. Acesso em: 10 jul. 2015.

BEAUDRY, C.; SCHIFFAUEROVA, A. Who's right, Marshall or Jacobs? The localization versus urbanization debate. Research Policy, v. 38, p. 318-337, 2009.

BETTENCOURT, L. M. A., LOBO, J., STRUMSKY, D. A. Invention in the city: increasing returns to patenting as a scaling function of metropolitan size. Research Policy, v. 36, n. 1, p. 107-120, 2007.

BRASIL. Ministério do Trabalho e Emprego. Relação Anual de Informações Sociais (RAIS). Brasília, DF: MTE, 2016.

BRENNER, T. Local industrial clusters: existence, emergence and evolution. [S. 1.]: Routledge, 2004. 
BRESCHI, S.; LISSONI, F. Mobility of skilled workers and co-invention networks: an anatomy of localized knowledge flows. Journal of Economic Geography, v. 9, n. 4, p. 439-468, July 2009.

BRESCHI, S.; LISSONI, F.; MALERBA, F. Knowledge-relatedness in firm technological diversification. Research Policy, v. 32, n. 1, p. 69-87, 2003.

CARLINO, G. A. Knowledge spillovers: cities' role in the new economy. Business Review, Philadelphia, v. 4, p. 17-26, 2001.

CHIARINI, T.; RAPINI, M. S.; SILVA, L. A. Access to knowledge and catch-up: exploring some intellectual property rights data from Brazil and South Korea. Science and Public Policy, v. 44, n. 1, p. 95-110, 2016.

COHEN, W.; LEVINTHAL, D. Innovation and learning: the two faces of RED. The Economic Journal, v. 99, n. 397, p. 569-596, 1989.

DURANTON, G.; PUGA, D. The micro-foundations of urban agglomeration economies. In: HENDERSON J. V.; THISSE J. F. (Eds.). Handbook of regional and urban economics. Amsterdam: Elsevier, 2004. p. 2063-2117. v. 4.

FELDMAN, M. P.; FLORIDA, R. The geographic sources of innovation: technological infrastructure and product innovation in the United States. Annals of the Association of American Geographers, v. 84, n. 2, p. 210-229, 1994.

FELDMAN, M. P.; KOGLER, D. F. Stylized facts in the geography of innovation. In: HALL, B. H.; ROSENBERG, N. (Ed.). Handbook of economics of innovation. [S. 1.]: Elsevier, 2010. p. 381-410.

FISCHER, M.; VARGA, A. Spatial knowledge spillovers and university: research: evidence from Austria. The Annals of Regional Science, v. 37, n. 2, p. 303-322, 2003.

FORAY, D.; LISSONI, F. University research and public-private interaction. In: HALL, B. H.; ROSENBERG, N. (Eds.). Handbook of the economics of innovation. [S. 1.]: Elsevier, 2010. p. 275-314. v. 1.

GLAESER, E. L. Learning in cities. Journal of Urban Economics, v. 46, n. 2, p. 254-277, 1999.

GONÇALVES, E. Estrutura urbana e atividade tecnológica em Minas Gerais. Economia Aplicada, Ribeirão Preto, v. 10, n. 4, p. 481-502, out./dez. 2006.

GONÇALVES, E.; ALMEIDA, E. Innovation and spatial knowledge spillovers: evidence from Brazilian patent data. Regional Studies, v. 43, p. 513-528, 2009.

GREUNZ, L. Geographically and technologically mediated knowledge spillovers between European regions. The Annals of Regional Science, v. 37, n. 4, p. 657-680, 2003.

HALL, B. H.; LERNER, J. The financing of RED and innovation. In: HALL, B. H.; ROSENBERG, N. (Eds.). Handbook of the economics of innovation, [S. 1.]: Elsevier, 2010. p. 609-639. v. 1. 
IBGE. Sistema IBGE de Recuperação Automática - SIDRA. Estimativas de População, população residente estimada. Disponível em: < https://sidra.ibge.gov.br/pesquisa/estimapop/ tabelas>. Acesso em: 10 maio 2016.

INSTITUTO NACIONAL DE PROPRIEDADE INDUSTRIAL - INPI. Base de Dados Estatísticos de Propriedade Intelectual (BADEPI). Rio de Janeiro, tabulação especial, 2012.

JACOBS, J. The economy of cities. Nova York: Random House, 1969.

JAFFE, A. Real effects of academic research. The American Economic Review, p. 957-970, 1989.

KRUGMAN, P. Confronting the mystery of urban hierarchy. Journal of the Japanese and International Economies, v. 10, n. 4, p. 399-418, 1996.

LOBO, J.; STRUMSKY, D. Metropolitan patenting, inventor agglomeration and social networks: a tale of two effects. Journal of Urban Economics, v. 63, n. 3, p. 871-884, 2008.

LUCAS, R. E. On the mechanics of economic development. Journal of Monetary Economics, v. 22, p. 3-42, 1988.

MALECKI, E. J. The geography of innovation. In: FISCHER, M. M.; NIJKAMP, P. (Eds.). Handbook of regional science. Berlin; Heidelberg: Springer; Verlag, 2014. p. 375-389. v. 1.

MARSHALL, A. Princípios de economia. São Paulo: Abril Cultural, 1982.

MIGUÉLEZ, E.; MORENO, R. Skilled labour mobility, networks and knowledge creation in regions: a panel data approach. The Annals of Regional Science, v. 51, n. 1, p. 191-212, 2013.

MUENDLER, M. A.; RAUCH, J. E. Mobilizing social capital through employee spinoffs. Cambridge, MA, US, 2012. (NBER Working Paper n. 18459).

Ó HUALLACHÁIN, B. Patent places: size matters. Journal of Regional Science, v. 39, p. 613636, 1999.

ORGANIZATION FOR ECONOMIC CO-OPERATION AND DEVELOPMENT - OECD. ISIC rev. 3 technology intensity definition: classification of manufacturing industries into categories based on RED intensities. Directorate for Science, Technology and Industry. Economic Analysis and Statistics Division, 2011.

ROMER, P. M. Increasing returns and long run growth. Journal of Political Economy, v. 94, p. 1002-1037, 1986.

ROSENTHAL, S.S., STRANGE, W.C. Evidence on the nature and sources of agglomeration economies, in: Henderson, V.; Thisse, J.F. (Eds.), Handbook of Regional and Urban Economics - Vol 4. pp. 2120-2167, 2004. doi:10.1016/S0169-7218(04)07049-2

RUIZ, R. M. Estruturas urbanas comparadas: Estados Unidos e Brasil. Estudos Econômicos, São Paulo, v. 35, n. 4, p. 715-737, 2005.

SCHERER, F. M. Inter-industry technology flows in the United States. Research Policy, v. 11, n. 4, p. 227-245, 1982. 
SIMMIE, J. Innovative cities. London: Spon Press, 2001.

VAN OORT, F. G.; LAMBOOY, J. G. Cities, knowledge and innovation. In: FISCHER, M. M.; NIJKAMP, P. (Eds.). Handbook of regional science. Berlin; Heidelberg: Springer; Verlag, 2014. p. 475-488. v. 1.

Recebido em: 16/05/2016. Aceito em: 09/04/2018. 